\section{Survival Functions}

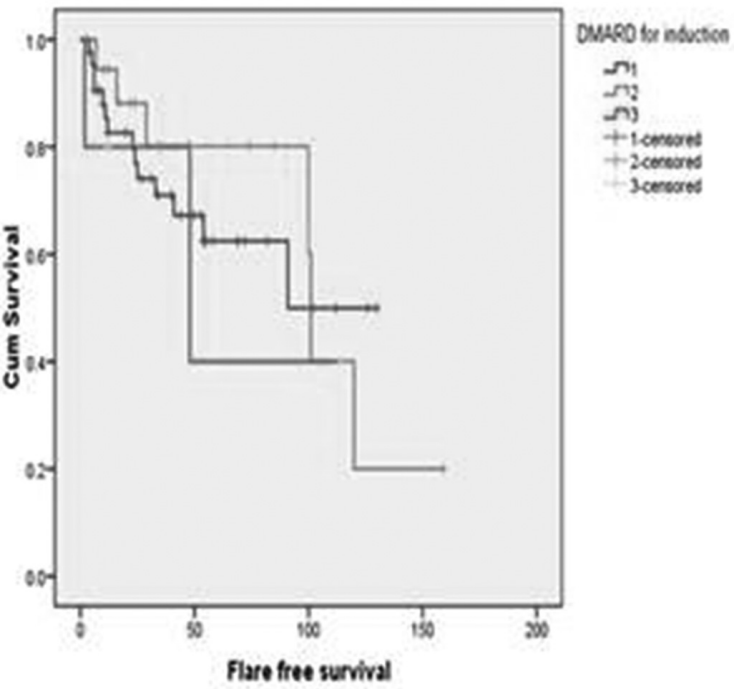

Abstract 124 Figure 1 Kaplan Meir survival curve: Median time to flare with Drug1 (MMf) 91 months as induction agent vs 101 months with drug 2(CYC) and 48 months with drug 3(Aza)

\section{OUTCOME OF CHILDHOOD LUPUS NEPHRITIS: A 15 YEARS EXPERIENCE OF A SINGLE CENTRECENTER}

${ }^{1} S$ Al Mayouf*, ${ }^{2}$ A Al Ameer, ${ }^{2}$ A Areej, ${ }^{2}$ A Alsonbul. 'Riyadh, Kingdom of Saudi Arabia; ${ }^{2}$ King Faisal Specialist Hospital, Paediatrics, Riyadh, Kingdom of Saudi Arabia

\subsection{6/lupus-2017-000215.125}

Background and aims To report the long-term renal outcome of a cohort of Saudi children with systemic lupus erythematosus (SLE)

Methods All patients with childhood lupus nephritis (cLN) proved by renal biopsy seen between January 2000 and June 2015 were reviewed The renal outcome was assessed according to serum creatinine level, protein/creatinine ratio at the last follow-up visit, and/or evidence of renal impairment during follow-up period and end stage renal disease (ESRD). Additional outcome measures include accrual damage measured by pSDI and death related to SLE was determined.

Results A total of 84 (72 females) cLN patients with followup duration of 9.3 years were included. The mean current age was 19.4 years and mean age at onset was 9.2 years. The most frequent histological class was proliferative glomerulonephritis (64.3\%) followed by membranous nephritis (27.4\%). The mean activity and chronicity were 6 and 4 respectively. Renal microthrombosis was found in 9 (10.7\%) patients. All patients treated with immunosuppressive medications; cyclophosphamide used in 64 followed by mycophenolate mofetil in 42 while rituximab used in 24 patients. At last follow up visit, the mean serum creatinine was 147 and the mean protein/creatinine ratio was 0.8 while the mean total SDI was 1.89. Sixteen (19\%) patients had ESRD. However, there was no significant difference in ESRD by histological class. The overall survival rates were 5 years: $94 \%$ and 10 years: $87 \%$. Infection was the leading cause of mortality.

Conclusions Our patients had severe cLN and required intensive treatment. However, the survival rate is comparable to other studies.

\section{AUTOIMMUNE HEMOLYTIC ANAEMIA EVOLVING TO SYSTEMIC LUPUS ERYTHREMATOSUS}

MY Anggara, B Setiabudiawan. Faculty Of Medicine Padjadjaran University/Hasan Sadikin General Hospital, paediatric, Bandung, Indonesia

\subsection{6/lupus-2017-000215.126}

Background and Aims The first manifestation of systemic lupus erythrematosus (SLE) could vary, atypical, and often confusing. Hemolytic anaemia can be the only manifestation of SLE. With low index of clinical suspicion or inadequate follow up, the diagnosis of SLE could be delayed. We present a case of autoimmune hemolytic anaemia (AIHA) patient to describe the nature of AIHA which could evolve to SLE.

Methods Case report of a 12-year-old Indonesian female was admitted to Dr. Hasan Sadikin General Hospital with complaints of pallor, chest pain, prolonged fever and redness on her cheek. She was diagnosed with idiopathic AIHA but never routinely checked up after being discharged from the hospital 6 months ago. On admission she was alert, febrile, had muffled heart sound, and malar rash. Her laboratory investigation revealed anaemia ( $\mathrm{Hb}: 6,8 \mathrm{~g} / \mathrm{dl}$ ), low $\mathrm{C} 3$ and $\mathrm{C} 4$, and increased titer of anti ds-DNA. Chest radiograph showed cardiomegaly and echocardiography showed pericardial effusion. A diagnosis of autoimmune hemolytic anaemia, systemic lupus erythrematosus, and pericardial effusion was confirmed. She was administered metilprednisolon $40 \mathrm{mg} /$ day.

Results The patient had good response to metilprdnisolon. Fever, malar rash, chest pain start to resolve at day 4th of steroid administration. She was discharged at day 7 th. One month after hospital discharge lupus activity disease remained stable and steroid was tappered off.

Conclusions We conclude that AIHA can be the only first symptoms of SLE. A careful observation of AIHA is important to prevent late diagnosis and treatment of SLE.

\section{CARDIOVASCULAR COMPLICATIONS IN PEDIATRIC- ONSET SYSTEMIC LUPUS ERYTHEMATOSUS IN SAUDI ARABIAN PATIENTS}

M Muzaffer, AS Azhar*. King Abdulaziz University Hospital, Department of paediatric, Jeddah, Saudi Arabia

\subsection{6/lupus-2017-000215.127}

Background and Aims Cardiac involvement among pSLE patients is a known complications. Early diagnosis and treatment of pSLE cardiac complications is crucial, as they may carry poor prognosis. We are reporting the prevalence and different types of cardiovascular complications in Saudi Arabian patients with pSLE.

Methods 46 pSLE patients (6 to 19 years) were following from January 2014 to September 2015 at the rheumatology clinic of King Abdul-Aziz University Hospital, Jeddah. Laboratory data such as CRP, ANA, anti-dsDNA, C3 and C4 complements, were collected. Cardiac evaluation included chest x-ray, ECG, and echocardiography, along with estimation of SLE activity by calculating the SLE Disease Activity Index (SLEDAI) score according to SELENA Modification

Results Prevalence of cardiac manifestations was $47.8 \%$, occurring at a mean $\pm \mathrm{SD}$ age of $14.0 \pm 2.28$ years. Valvular heart diseases were detected in $16(34.8 \%)$ cases, followed by pericarditis in $6(13 \%)$, and silent valvular diseases in $8(17.4 \%)$ cases. Of the 16 valvular diseases, tricuspid and pulmonary 